\title{
A Semiempirical MIMO Channel Model in Obstructed Viaduct Scenarios on High-Speed Railway
}

\author{
Tao Zhou, ${ }^{1}$ Cheng Tao, ${ }^{1}$ Liu Liu, ${ }^{1,2}$ and Zhenhui Tan ${ }^{1}$ \\ ${ }^{1}$ Institute of Broadband Wireless Mobile Communications, Beijing Jiaotong University, Beijing 100044, China \\ ${ }^{2}$ National Mobile Communications Research Laboratory, Southeast University, Nanjing 210096, China \\ Correspondence should be addressed to Tao Zhou; 11111037@bjtu.edu.cn
}

Received 13 November 2013; Revised 14 April 2014; Accepted 17 April 2014; Published 12 June 2014

Academic Editor: Selvan T. Krishnasamy

Copyright (c) 2014 Tao Zhou et al. This is an open access article distributed under the Creative Commons Attribution License, which permits unrestricted use, distribution, and reproduction in any medium, provided the original work is properly cited.

\begin{abstract}
A semiempirical multiple-input multiple-output (MIMO) channel model is proposed for high-speed railway (HSR) viaduct scenarios. The proposed MIMO model is based on the combination of realistic single-input single-output (SISO) channel measurement results and a theoretical geometry-based stochastic model (GBSM). Temporal fading characteristics involving $K$ factor and Doppler power spectral density (PSD) are derived from the wideband measurement under an obstructed viaduct on Zhengzhou-Xian HSR in China. The GBSM composed of a one-ring model and an elliptical model is employed to describe the entire propagation environment. Environment-related parameters in the GBSM are determined by the measured temporal fading properties. And a close agreement is achieved between the model results and measured data. Finally, a deterministic simulation model is established to perform the analysis of the space-time correlation function, the space-Doppler PSD, and the channel capacity for the measured scenario. This model is more realistic and particularly beneficial for the performance evaluation of MIMO systems in HSR environments.
\end{abstract}

\section{Introduction}

Public communications on high-speed railway (HSR) are currently attracting extensive attention all over the world. Train passengers expect to enjoy high-quality data communication services as they do on the ground. However, present HSR communication systems used for train management and safety are difficult to satisfy their demands. To achieve a better user experience on HSR, a tailor-made communication service system for passengers needs to be researched and developed. In addition, some advanced technologies such as multiple-input multiple-output (MIMO) and relay should also be applied to HSR communication systems to enhance channel capacity and transmission reliability. In general, channel models are important for optimization, test, and performance evaluation of communication systems as well as related technologies. As HSR propagation scenarios determine unique channel fading characteristics, it is therefore also of importance to develop reasonable HSR channel models to facilitate researches on HSR communications.
Owing to the special nature of HSR such as high speed mobility and multiple propagation scenarios, it is rather difficult to carry out field measurements and acquire sufficient channel data. Compared with traditional terrestrial cellular environments, for example, urban, suburban, and rural micro- and macrocell, studies on the channel models under the HSR scenarios are inadequate. The existing HSR channel models are mainly classified into three categories as follows.

(1) Standardized Model. Third Generation Partnership Project (3GPP) defines a nonfading channel model for the test of the baseband performance on high-speed train conditions in technical specification (TS) 36.104 [1]. This model identifies an essential Doppler feature that the receiver encounters a rapid Doppler transition when the train passes through base station (BS). Furthermore, the WINNER II model [2] details both large-scale and small-scale fading characteristics of fast train measurements in its moving networks (D2a) scenario where the mobile relay technology 
is considered, consisting of two channels, BS-mobile relay station (MRS) and MRS-mobile station (MS).

(2) Measurement-Based Model. This type of model is mainly based on the channel measurement results in typical HSR scenarios such as tunnel, viaduct, and U-shape cutting. HSR tunnel wideband measurements at $2.1 \mathrm{GHz}$ are conducted by Helsinki University of Technology in France and Belgium [3]. Channel parameters like path loss (PL), delay, and Doppler are characterized. Authors in $[4,5]$ have performed extensive narrowband measurements on HSR at $930 \mathrm{MHz}$. Empirical $\mathrm{PL}$ and $K$-factor models with respect to the viaduct height and $U$-shape cutting width are originally presented. Liu et al. [6] especially have proposed a position-based wideband HSR model at $2.35 \mathrm{GHz}$, which is based on the channel data collected under a viaduct scenario.

(3) Theory-Based Model. Geometry and random-propagation-graph theories are two feasible approaches for radio channel modeling. References [7, 8] establish a geometrybased stochastic model (GBSM) and use it to analyze the space-time-frequency correlation and nonstationary properties of HSR channels. In [9], a graph-based simulation model is developed to characterize time-variant channels for HSR communications.

In fact, there are still no realistic MIMO channel models for the typical HSR scenarios. Although the standardized model has provided spatial characteristics of the HSR channel, it may not be applicable in realistic viaduct or U-shape cutting scenarios. As for the measurement-based models, they mainly focus on the single-input single-output (SISO) channel properties since MIMO channel measurements on HSR are currently hard to implement. The theory-based models are highly suitable for the analysis and simulation of the MIMO channel, whereas the present researches are theoretical and lack support of actual measurement data on HSR.

The goal of this paper is to develop the MIMO channel model for the typical HSR scenarios. Rather than trying to measure the MIMO channel or merely deducing the theoretical MIMO model, this study is based on the combination of SISO channel measurement results and the GBSM. Temporal fading characteristics involving $K$-factor and Doppler power spectral density (PSD) are derived from channel measurements in an obstructed viaduct scenario on Zhengzhou-Xian HSR in China. The GBSM that consists of a one-ring model and an elliptical model is employed to describe the measured propagation environment. According to a nonlinear least squares method, environment-related parameters in the GBSM are determined by the temporal fading characteristics. By applying the environment-related parameters to the GBSM and using a parameter computation method, a semiempirical MIMO channel simulation model is established. Finally, based on the proposed model, we perform the analysis of the space-time (ST) correlation function (CF), space-Doppler (SD) PSD, and channel capacity for the measured viaduct scenario.

The remainder of this paper is outlined as follows. Section 2 describes the channel measurements and results.
In Section 3, a semiempirical MIMO channel model is established. Simulation results and analysis are presented in Section 4. Finally, conclusions are drawn in Section 5.

\section{Channel Measurements and Results}

2.1. Measurement Description. A standard radio channel sounder, Propsound by Elektrobit, is employed to perform wideband measurements on Zhengzhou-Xi'an HSR in China. It consists of a dedicated transmitter and receiver. The simplified block schematic is shown in Figure 1. Basically, the transmitter consists of a control notebook, a code generator, an up converter, and a transmit antenna. The measuring receiver is made up of a receive antenna, a down converter, a data acquisition and storage unit, and a real-time display and control notebook. At the transmitter, a binary m sequence with the length of 127 is periodically transmitted by a vertical polarized dipole antenna. The chosen measurement frequency and bandwidth are set to $2.35 \mathrm{GHz}$ and $50 \mathrm{MHz}$, respectively. The transmit power is $30.8 \mathrm{dBm}$. The relay link from transmitter to MRS is adopted. The MRS antenna, HUBER + SUHNER [10], is positioned on the top of the train. Channel impulse responses (CIRs) are recovered by correlating the received signal with a sequence copy identical to the one used at the transmitter. The channel sample rate is set to $1968.5 \mathrm{~Hz}$. Clocks between the transceivers are synchronized by the GPS network which provides an accurate universal timing signal.

The viaduct is one of the most typical HSR scenarios [11]. Our measured viaduct scenario and corresponding surroundings are shown in Figure 2. The transmit antenna is elevated about $4.8 \mathrm{~m}$ and placed on the roof of a farm house ( $8 \mathrm{~m}$ height), which approximates $92 \mathrm{~m}$ away from the viaduct ( $8 \mathrm{~m}$ height). The receive antenna is about $3.8 \mathrm{~m}$ higher than the rail. The average velocity of the train is $198 \mathrm{~km} / \mathrm{h}$. Some local scatterers are found along the viaduct, which can be divided into two categories, short sparse trees and high dense trees. The high dense trees especially cause a strong obstruction of the radio propagation between the transceivers. However, the distribution of these scatterers is not continuous; thus, the $\mathrm{Rx}$ encounters both line-of-sight (LOS) and non-line-of-sight (NLOS) propagation states in the entire measurement environment.

2.2. Data Processing. The measurement record consists of $N=59862$ CIRs corresponding to $30.4 \mathrm{sec}$ of continuous channel data. Each CIR with the delay resolution of $0.2 \mu \mathrm{s}$ contains $M=254$ multipath taps. The raw CIRs can be denoted by a $N \times M$ complex matrix, $h(n, \tau)$, where $n$ is the CIR number and $\tau$ is the multipath tap index. In this paper, we mainly concentrate on the modeling of narrowband channels. Thus, we transform the wideband CIRs into the narrowband form. The complex sum of $h(n, \tau)$ is made over the delay domain according to

$$
h(n)=\int h(n, \tau) \mathrm{d} \tau .
$$




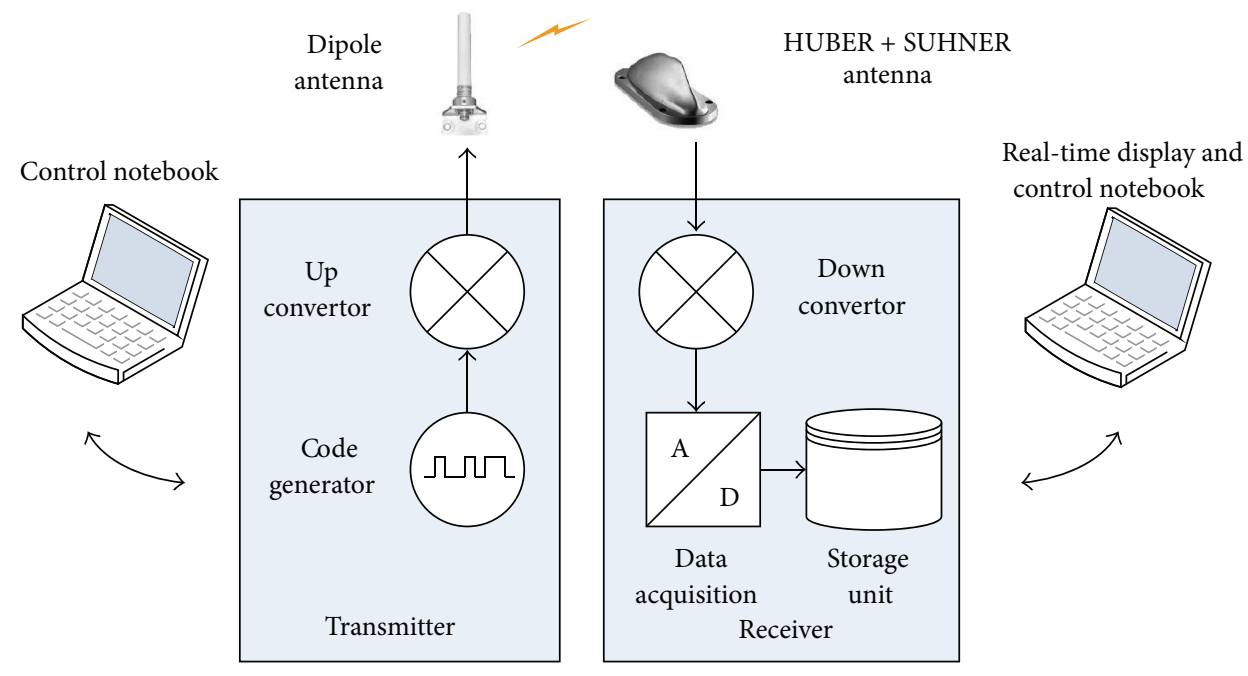

Figure 1: Propsound block schematic.
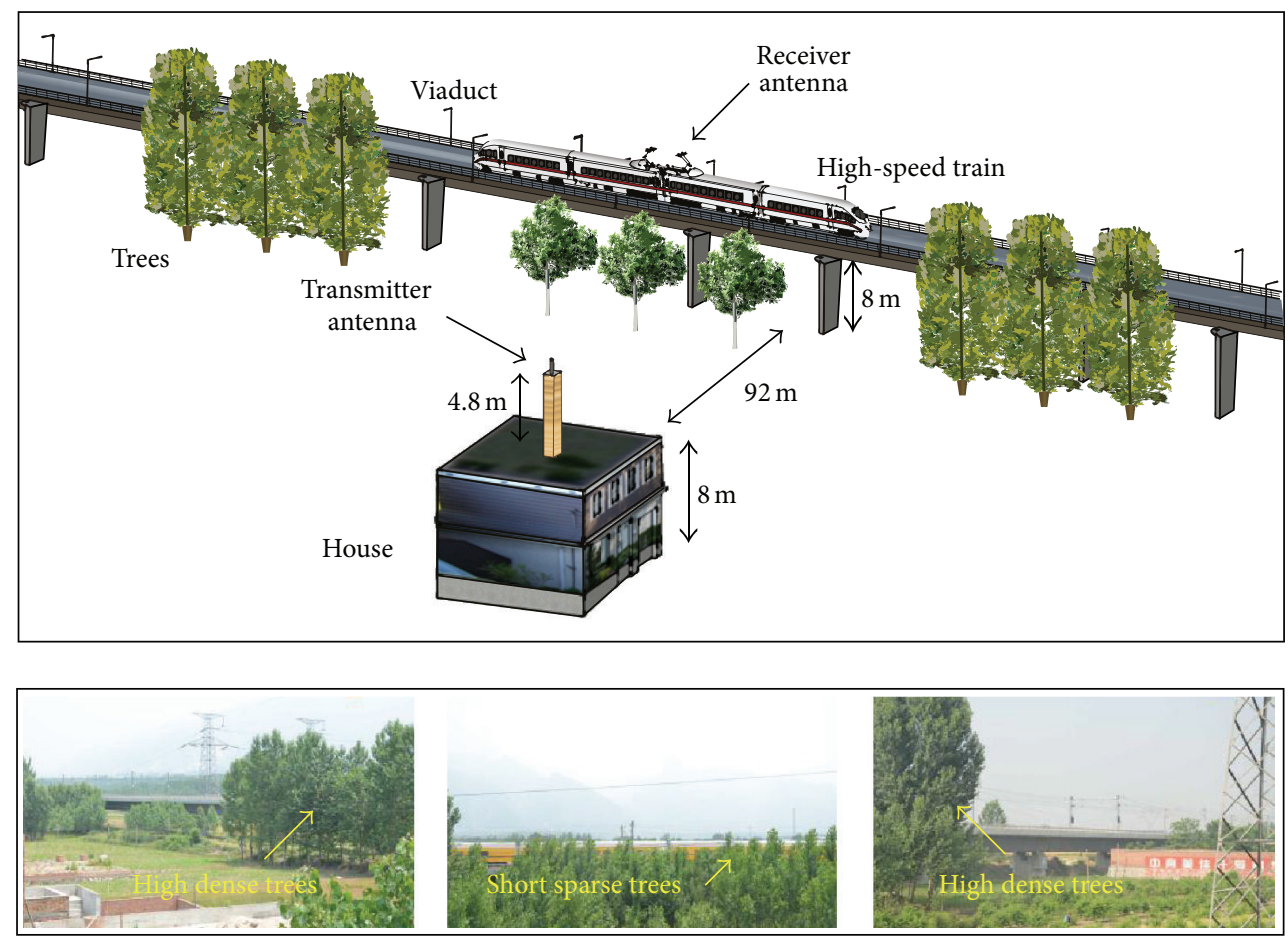

FIGURE 2: The measured viaduct scenario and corresponding surroundings.

Then, we remove the large-scale fading effect including the PL and shadowing to normalize the narrowband CIRs, expressed as

$$
h^{\prime}(n)=\frac{h(n)}{\sqrt{P_{a}(n)}},
$$

where $P_{a}(n)$ denotes the average power of CIRs, which can be calculated by a sliding window with the length of $W$ according to

$$
P_{a}(n)=\frac{1}{W} \sum_{k=n}^{n+W-1}|h(k)|^{2}
$$




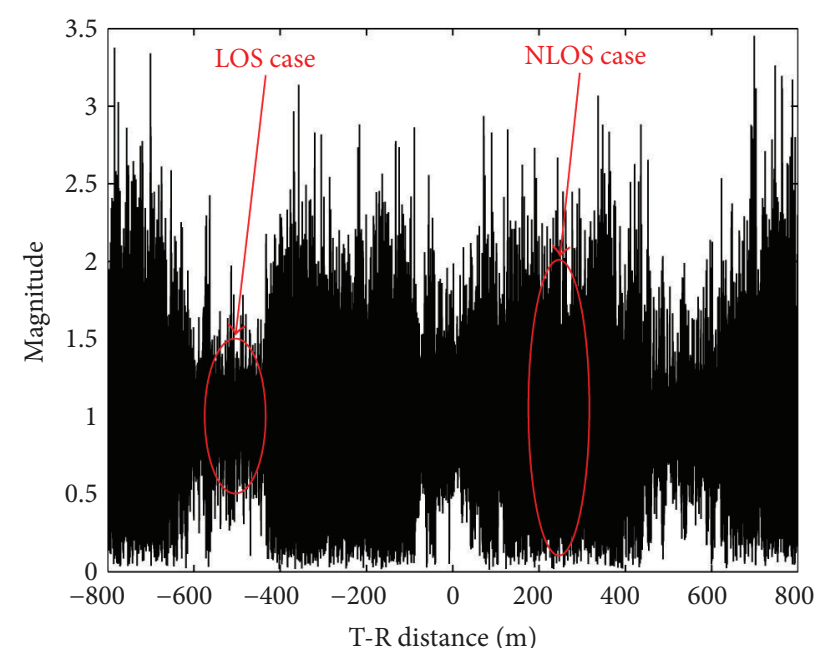

FIgURE 3: Measurement envelope.

$W$ is recommended to 10 wavelengths with the distance of $1.28 \mathrm{~m}$ at $2.35 \mathrm{GHz}[12]$.

A linear plot of $\left|h^{\prime}(n)\right|$ is shown in Figure 3. It is observed that the envelope fluctuates dramatically in some regions, while it is fairly concentrated around the mean in other areas. This confirms the fact that there are different propagation conditions including LOS and NLOS in the entire scenario. The LOS case stabilizes the envelope variation. The NLOS case, however, aggravates the envelope fluctuation.

2.3. Temporal Fading Characteristics. $K$-factor and Doppler PSD are two temporal fading parameters that, respectively, measure how much and how fast local fading the signal experiences over time. The temporal fading characteristics are the key of channel modeling as well. Based on the processed CIRs, we can extract the $K$-factor and the Doppler PSD.

$K$-factor is usually defined as the power ratio of fixed components to scatter components in the received signal. When $K$-factor is infinite, there is no fading; otherwise, there is Ricean fading $(K>0)$ or Rayleigh fading $(K=$ $0)$. According to [13], a moment-based method is used to estimate the time-variant $K$-factor as follows:

$$
\widehat{K}(n)=\frac{\sqrt{E^{2}\left\{P_{h}(n)\right\}-\operatorname{Var}\left\{P_{h}(n)\right\}}}{E\left\{P_{h}(n)\right\}-\sqrt{E^{2}\left\{P_{h}(n)\right\}-\operatorname{Var}\left\{P_{h}(n)\right\}}},
$$

where $P_{h}(n)=\left|h^{\prime}(n)\right|^{2}$ and $E(\cdot)$ and $\operatorname{Var}(\cdot)$ denote the expectation and variance, respectively.

Figure 4 shows results of the $K$-factor over T-R distance. The T-R distance represents relative horizontal distance between the transceivers. The positive T-R distance indicates the train moving away from the $\mathrm{Tx}$, while the negative denotes the train running towards the Tx. It can be seen that there is a good match between Figures 3 and 4. The high $K$-factor is observed in the LOS regions where the envelope fluctuates slightly, whereas the low $K$-factor is found in the NLOS regions in which the envelope changes in a wider

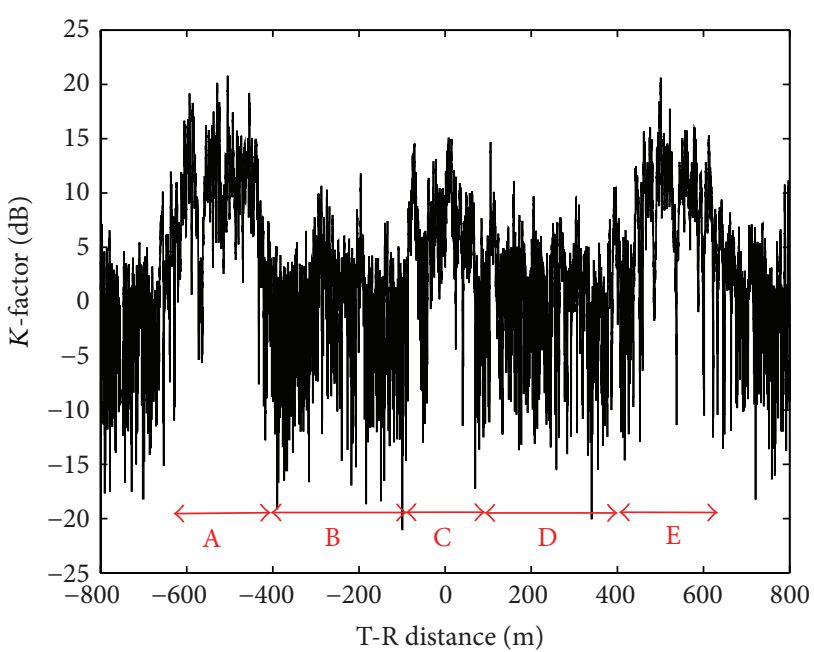

Figure 4: The $K$-factor against T-R distance.

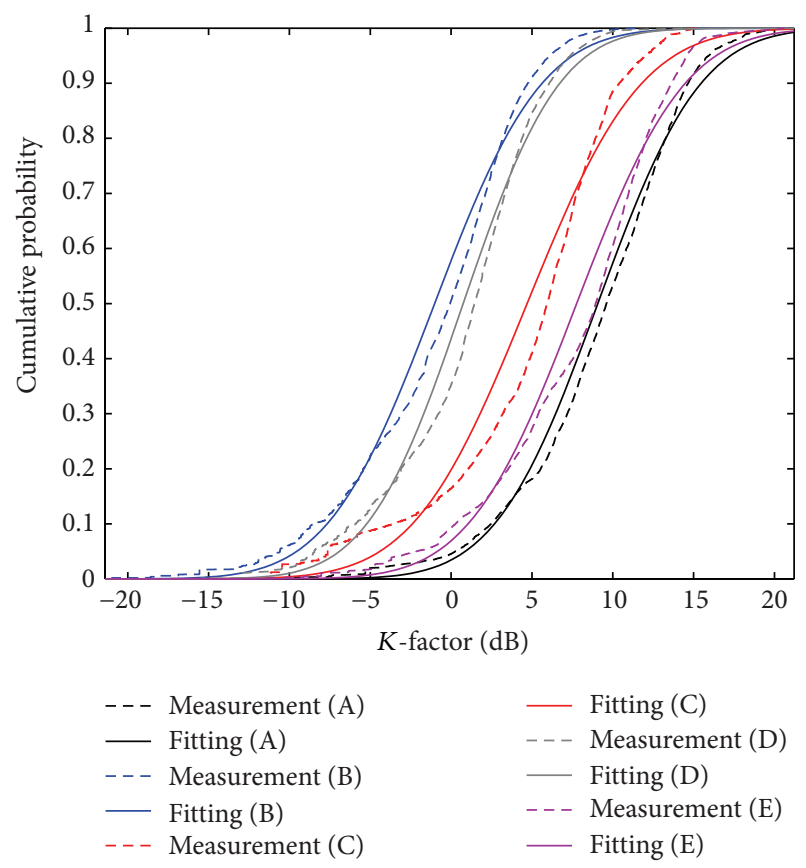

Figure 5: CDFs of the $K$-factor in different propagation segments.

range. According to the $K$-factor results, we can make a clear propagation segmental partition for the whole scenario, as listed in Table 1. The fitting results by Gaussian distribution for different propagation segments are shown in Figure 5. It is found that the $K$-factor in the $\mathrm{C}$ segment is lower than that in the $\mathrm{A}$ and $\mathrm{E}$ segments. This is because there are the short sparse trees in the $\mathrm{C}$ segment producing some reflection and scattering waves that reduce the effect of LOS components. With respect to the NLOS segments such as B and D, the $K$ factor strength is close to zero due to the impact of a strong shadowing caused by the high dense trees.

Doppler PSD is a second-order statistic of the temporal fading. The movement of the MS or scatterers results in the 
TABLE 1: The $K$-factor results in different propagation segments.

\begin{tabular}{lccccc}
\hline & & \multicolumn{2}{c}{ Propagation state } & \multicolumn{2}{c}{ NLOS } \\
Partitioned segment & A & LOS & E & $-95 \sim 95$ & $95 \sim 416$ \\
\hline Coverage range $[\mathrm{m}]$ & $-662 \sim-433$ & $-433 \sim-95$ & 7.52 & -1.02 & $416 \sim 644$ \\
Mean value $[\mathrm{dB}]$ & 9.08 & 4.71 & 5.24 & 0.75 \\
Standard variance $[\mathrm{dB}]$ & 4.98 & 5.53 & & 5.18 \\
\hline
\end{tabular}

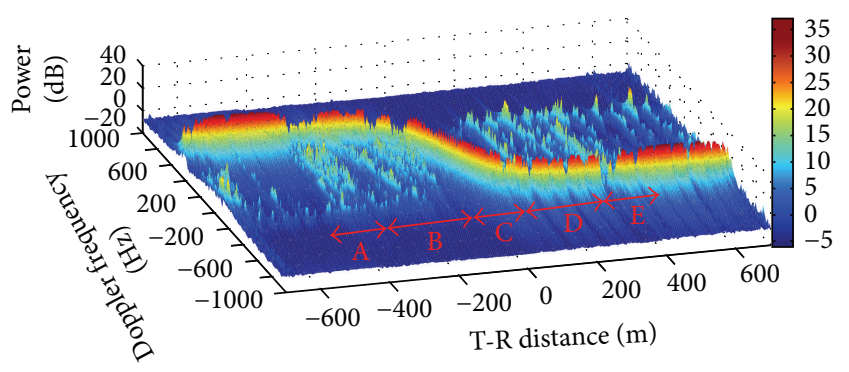

FIGURE 6: The instantaneous Doppler PSD over T-R distance.

Doppler frequency shift of multipath components. Doppler frequency shift is associated with the angle of arrival (AOA) between the multipath wave and direction of motion. Since multipath components with different AOAs have different Doppler frequency shifts, this causes a spreading of the received signal in the frequency domain. Doppler PSD can be calculated by the inverse Fourier transform of the autocorrelation function of $h^{\prime}(n)$, written as

$$
\widehat{S}(f)=\mathrm{fft}\left[\operatorname{corr}\left(h^{\prime}(n)\right), L\right],
$$

where $\mathrm{fft}$ is the fast Fourier transformation, $L$ is the length of $\mathrm{ft}$, corr is the correlation operation, and $f$ is the Doppler frequency.

Figure 6 illustrates the instantaneous Doppler PSD over the T-R distance. It is obvious that the Doppler frequency shift has a fast variation from a positive maximum Doppler frequency to a negative one when the Rx locates in the segment C. This phenomenon is consistent with the result in [1]. Besides, we also find that the Doppler PSD in the $\mathrm{B}$ and $\mathrm{D}$ segments shows a certain spread, while the shape of the Doppler PSD is not like a classical JAKES spectrum. This confirms that the $\mathrm{B}$ and $\mathrm{D}$ segments are under the nonisotropic scattering environment. In contrast, there is almost no Doppler spread in the A and E segments because of the strong LOS and less scattering components and the Doppler PSD is close to a single spectrum line. In fact, the shape of the Doppler PSD is mainly determined by the angular distribution [14]. Thus, it is possible to obtain angular characteristics if we can understand the Doppler PSD of the measured channel.

\section{A Semiempirical MIMO Channel Model}

In this section, according to the combination of the temporal fading results and a theoretical GBSM, we establish a semiempirical MIMO channel model for the measured HSR viaduct scenario.

3.1. The Reference Model. We consider a narrowband $2 \times$ 2 MIMO communication system. Figure 7 illustrates the geometrical model, which involves a one-ring model [15] and an elliptical model [16]. The one-ring model is used to describe the obstructed environment such as the $\mathrm{B}$ and $\mathrm{D}$ segments, in which the transmitter is surrounded by local scatterers $S_{n_{1}}\left(n_{1}=1,2, \ldots, N_{1}\right)$. For the elliptical model, it is developed to represent the unobstructed environment like the $\mathrm{A}$ and $\mathrm{E}$ segments, where effective scatterers $S_{n_{2}}\left(n_{2}=\right.$ $1,2, \ldots, N_{2}$ ) are distributed on an ellipse with the transmitter and the receiver located at the focal points. Note that the $\mathrm{C}$ segment is not considered here. Transmit and receive antenna element spacings are denoted by $\delta_{T}$ and $\delta_{R}$, respectively. The distance between the transceivers is $D=2 f$ with $f$ indicating the half-length of the distance between the two focal points. The tilt angles of the array are denoted by $\beta_{T}$ and $\beta_{R}$. The angle of motion $\alpha_{v}$ indicates the angle between the $x$-axis and the direction of motion with the speed of $v$. The AOA and angle of departure (AOD) of the $n$th transmitted wave are designated as $\alpha_{n_{i}}^{R}$ and $\alpha_{n_{i}}^{T}, i=1,2$. Lastly, $\alpha_{\mathrm{LOS}}^{R}$ denotes the AOA of a direct ray.

The reference model based on the assumption that the number of scatterers is infinite can be derived from the presented geometrical model. The complex channel envelope between link $A_{1}^{T}$ and $A_{1}^{R}$ consists of the LOS and singlebounced diffuse components, which can be expressed as

$$
h_{11}(t)=h_{11}^{\mathrm{LOS}}(t)+h_{11}^{\mathrm{DIF}_{i}}(t)
$$

where

$$
\begin{aligned}
& h_{11}^{\mathrm{LOS}}(t)=\sqrt{\frac{\Omega_{11} K_{11}}{K_{11}+1}} e^{-(j 2 \pi / \lambda) D_{11}+j 2 \pi f_{D}\left[\cos \left(\alpha_{\mathrm{LOS}}^{R}-\alpha_{\nu}\right)\right] t}, \\
& h_{11}^{\mathrm{DIF}_{i}}(t)=\sqrt{\frac{\Omega_{11}}{K_{11}+1}} \lim _{N_{i} \rightarrow \infty} \frac{1}{\sqrt{N_{i}}} \sum_{n_{i}=1}^{N_{i}} e^{j \theta_{n_{i}}} \\
& \times e^{-(j 2 \pi / \lambda)\left(D_{1 n_{i}}^{T}+D_{n_{i}}^{R}\right)} e^{j 2 \pi f_{D}\left[\cos \left(\alpha_{n_{i}}^{R}-\alpha_{v}\right)\right] t} .
\end{aligned}
$$

In (7)-(8), $\Omega_{11}$ denotes the total power of the $A_{1}^{T}$ - $A_{1}^{R}$ link, $K_{11}$ indicates the $K$-factor, $\lambda$ is the wavelength, and $f_{D}$ 


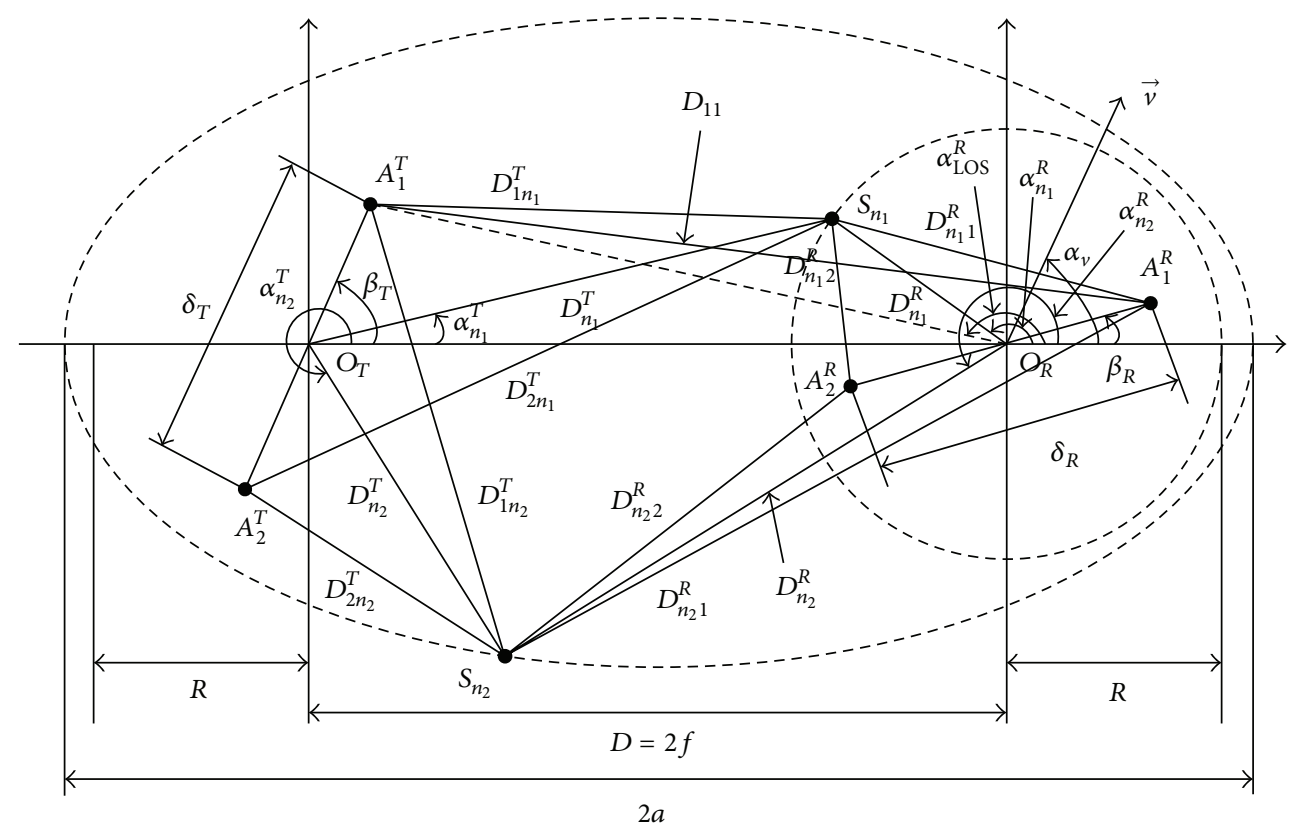

Figure 7: The geometrical model for a $2 \times 2$ MIMO channel.

represents the maximum Doppler frequency. The phase shift $\theta_{n_{i}}$ is distributed (i.i.d.) random variables with uniform distributions over $[-\pi, \pi]$.

From Figure 7 and using the law of cosines, the path length can be approximately given as

$$
\begin{gathered}
D_{11} \approx D-\frac{\delta_{T}}{2} \cos \beta_{T}-\frac{\delta_{R}}{2} \cos \left(\alpha_{\mathrm{LOS}}^{R}-\beta_{R}\right), \\
D_{1 n_{i}}^{T} \approx D_{n_{i}}^{T}-\frac{\delta_{T}}{2} \cos \left(\alpha_{n_{i}}^{T}-\beta_{T}\right), \\
D_{n_{i} 1}^{R} \approx D_{n_{i}}^{R}-\frac{\delta_{R}}{2} \cos \left(\alpha_{n_{i}}^{R}-\beta_{R}\right),
\end{gathered}
$$

where $D_{n_{1}}^{T}=\left(D^{2}+R^{2}+2 D R \cos \alpha_{n_{1}}^{R}\right)^{1 / 2}, D_{n_{2}}^{T}=\left(a^{2}+f^{2}+\right.$ $\left.2 a f \cos \alpha_{n_{2}}^{R}\right) /\left(a+f \cos \alpha_{n_{2}}^{R}\right), D_{n_{1}}^{R}=R$, and $D_{n_{2}}^{R}=b^{2} /(a+$ $\left.f \cos \alpha_{n_{2}}^{R}\right)$. By using the method proposed in [17], we can determine the relationship between the AOA and the AOD. Thus, $\alpha_{n_{i}}^{T}$ is expressed in terms of $\alpha_{n_{i}}^{R}$ as

$$
\begin{aligned}
\sin \alpha_{n_{1}}^{T} & =\frac{R \sin \alpha_{n_{1}}^{R}}{\sqrt{D^{2}+R^{2}+2 D R \cos \alpha_{n_{1}}^{R}}}, \\
\cos \alpha_{n_{1}}^{T} & =\frac{D+R \cos \alpha_{n_{1}}^{R}}{\sqrt{D^{2}+R^{2}+2 D R \cos \alpha_{n_{1}}^{R}}}, \\
\sin \alpha_{n_{2}}^{T} & =\frac{b^{2} \sin \alpha_{n_{2}}^{R}}{a^{2}+f^{2}+2 a f \cos \alpha_{n_{2}}^{R}}, \\
\cos \alpha_{n_{2}}^{T} & =\frac{2 a f+\left(a^{2}+f^{2}\right) \cos \alpha_{n_{2}}^{R}}{a^{2}+f^{2}+2 a f \cos \alpha_{n_{2}}^{R}} .
\end{aligned}
$$

3.2. ST CF and SD PSD. The ST CF between the two links $h_{11}(t)$ and $h_{22}(t)$ can be defined as [18]

$$
\begin{aligned}
\rho_{11,22}\left(\delta_{T}, \delta_{R}, \tau\right) & =\frac{E\left[h_{11}(t) h_{22}^{*}(t+\tau)\right]}{\sqrt{\Omega_{11} \Omega_{22}}} \\
& =\rho_{11,22}^{\mathrm{LOS}}\left(\delta_{T}, \delta_{R}, \tau\right)+\rho_{11,22}^{\mathrm{DIF}_{i}}\left(\delta_{T}, \delta_{R}, \tau\right),
\end{aligned}
$$

where $(\cdot)^{*}$ denotes the complex conjugation.

Assume that $\alpha_{\mathrm{LOS}}^{R}=\pi$ and $K_{11}=K_{22}=K_{i}$ and, substituting (7) and (9) into (13), we can obtain the ST CF of the LOS component as

$$
\rho_{11,22}^{\mathrm{LOS}}(\tau)=\frac{K_{i}}{K_{i}+1} e^{-(j 2 \pi / \lambda)\left(D_{11}-D_{22}\right)+j 2 \pi f_{D}\left[\cos \left(\alpha_{v}\right)\right] \tau} .
$$

Similarly, substituting (8), (10), and (11) into (13), we can express the ST CF of the single-bounced diffuse components as

$$
\begin{aligned}
\rho_{11,22}^{\mathrm{DIF}_{i}}\left(\delta_{T}, \delta_{R}, \tau\right)=\frac{1}{K_{i}+1} \int_{-\pi}^{\pi} & e^{-j 2 \pi f_{D}\left[\cos \left(\alpha_{n_{i}}^{R}-\alpha_{v}\right)\right] \tau} \\
& \times e^{-(j 2 \pi / \lambda)\left(D_{1 n_{i}}^{T}-D_{2 n_{i}}^{T}+D_{n_{i} 1}^{R}-D_{n_{i} 2}^{R}\right)} \\
& \times p\left(\alpha_{n_{i}}^{R}\right) \mathrm{d} \alpha_{n_{i}}^{R},
\end{aligned}
$$

where $p\left(\alpha_{n_{i}}^{R}\right)$ denotes the probability distribution function (PDF) of AOA. In this paper, we employ the von Mises PDF [14], which is very suitable for describing the distribution of local scatterers in the nonisotropic scattering environment. Thus, the PDF of AOA is expressed as $p\left(\alpha_{n_{i}}^{R}\right)=$ $e^{\kappa_{i} \cos \left(\alpha_{n_{i}}^{R}-\mu_{i}\right)} / 2 \pi I_{0}\left(\kappa_{i}\right)$, where $\alpha_{n_{i}}^{R} \in[-\pi, \pi), I_{0}(\cdot)$ is the zerothorder modified Bessel function of the first kind, $\mu_{i}$ is the mean 


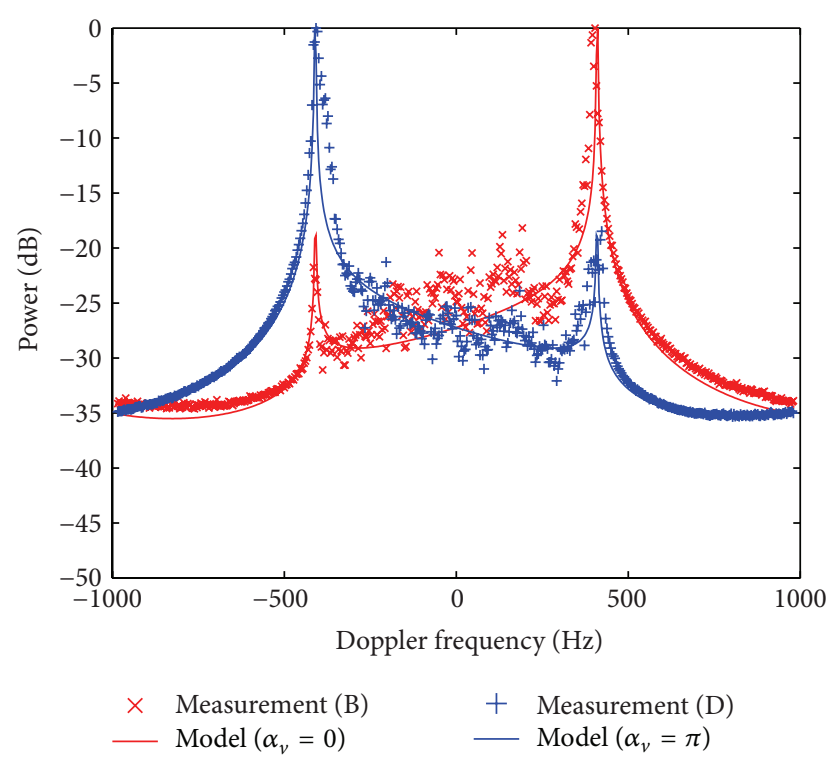

Figure 8: Doppler PSDs of the measurement and the proposed model for the obstructed environment $\left(\delta_{T}=\delta_{R}=0\right)$.

value of $\alpha_{n_{i}}^{R}$, and $\kappa_{i}$ is a parameter controlling the angular spread of $\alpha_{n_{i}}^{R}$.

Applying the Fourier transform to the ST CF in (13) in terms of $\tau$, we can derive the corresponding SD PSD as

$$
\begin{aligned}
S_{11,22}\left(\delta_{T}, \delta_{R}, f\right) & =\int_{-\infty}^{\infty} \rho_{11,22}\left(\delta_{T}, \delta_{R}, \tau\right) e^{-j 2 \pi f_{d} \tau} \mathrm{d} \tau \\
& =S_{11,22}^{\mathrm{LOS}}\left(\delta_{T}, \delta_{R}, f\right)+S_{11,22}^{\mathrm{DIF}_{i}}\left(\delta_{T}, \delta_{R}, f\right) .
\end{aligned}
$$

3.3. The Semiempirical Deterministic Simulation Model. According to (16), the Doppler PSD for the SISO case can be obtained by setting $\delta_{T}=\delta_{R}=0$, which is mainly associated with the environment-related parameters such as $\kappa_{i}, \mu_{i}$, and $K_{i}$. Based on the measured temporal fading characteristics, the nonlinear least squares method is employed to estimate the angular parameters $\left(\kappa_{i}, \mu_{i}\right)$ in the GBSM as follows:

$$
\left(\kappa_{i}, \mu_{i}\right)=\underset{\left(\kappa_{i}, \mu_{i}\right)}{\arg \min }\left\{\frac{1}{L} \sum_{l=1}^{L}\left[\widehat{S}\left(f_{l}\right)-S_{11,22}\left(f_{l}\right)\right]^{2}\right\} .
$$

As for the obstructed segment, we regard it as the complete NLOS case, thus setting $K_{1}=0$. Depending on the $K$-factor results as listed in Table 1 , we choose $K_{2}=8.3 \mathrm{~dB}$ for the unobstructed segment, which is calculated by averaging the mean value of the $K$-factor in the $\mathrm{A}$ and $\mathrm{E}$ segments. From (17), the fitting results are given as $\kappa_{1}=1.1, \mu_{1}=137.6^{\circ}$, $\kappa_{2}=7.2$, and $\mu_{2}=22.9^{\circ}$. It is reasonable that $\kappa_{1}$ is lower than $\kappa_{2}$ because the rich multipath components in the obstructed environment cause the larger angular spread. As for $\mu_{1}$ and $\mu_{2}$, they are related to the location of the scatterers.

Figures 8 and 9 depict the measurement and model results for the Doppler PSD in the obstructed and unobstructed environments. Note that the model results are obtained by

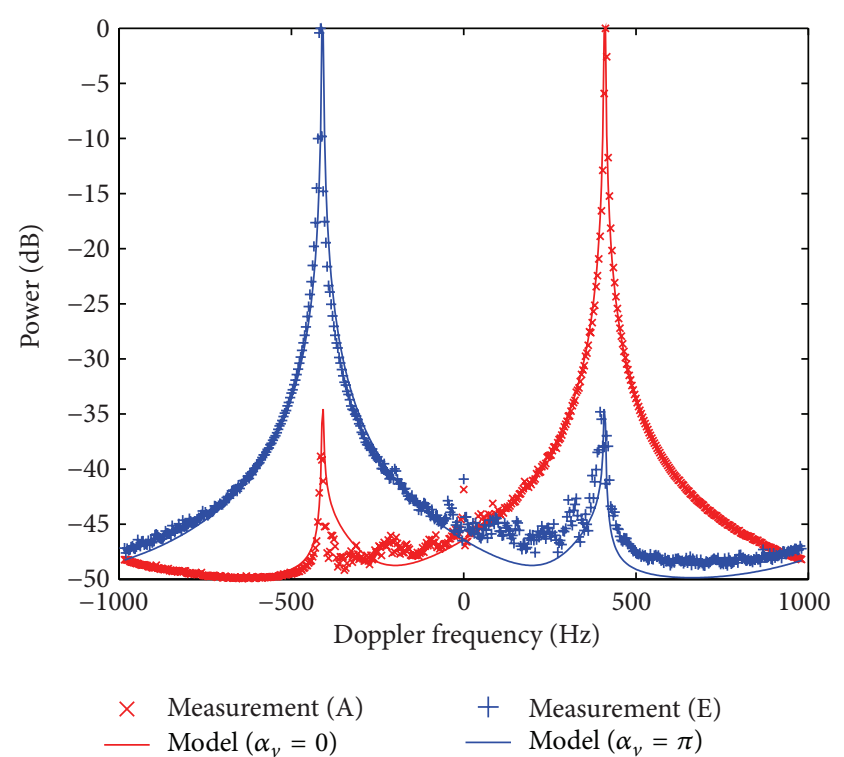

FIGURE 9: Doppler PSDs of the measurement and the proposed model for the unobstructed environment $\left(\delta_{T}=\delta_{R}=0\right)$.

employing the same environment-related parameters but the opposite angle of motion. We can find that there is a good match between the measurement and model results in both environments. Thus, by applying the environment-related parameters to the reference model, we can extend the model results in the SISO case to those in the MIMO case by setting $\delta_{T} \neq 0$ and $\delta_{R} \neq 0$.

Besides, a deterministic simulation model should be derived from the reference model by setting a finite number of scatterers $N_{i}$ and the fixed phases $\theta_{n_{i}}$ since the reference model cannot be used to implement the simulation. The discrete AOAs of scatterers are constants in the simulation model, which must provide a good approximation of autocorrelation function (ACF) to the reference model. The modified method of equal areas (MMEA) [19] is a low complexity but high accuracy parameter computation method for nonisotropic channels. We employ MMEA algorithm to compute the AOAs as

$$
\alpha_{n_{i}}^{R}=F_{\alpha}^{-1}\left[\frac{1}{N_{i}}\left(n_{i}-\frac{1}{4}\right)\right]
$$

where $F_{\alpha}^{-1}(\cdot)$ denotes the inverse function of $F_{\alpha}(\cdot)$ that is the cumulative distribution function (CDF) of the AOAs.

\section{Simulation Results and Analysis}

In this section, based on the proposed MIMO simulation model, the ST CF, SD PSD, and channel capacity are analyzed for the unobstructed and obstructed segments of the viaduct scenario. The parameters of the model are set as $f_{c}=$ $2.35 \mathrm{GHz}, v=198 \mathrm{~km} / \mathrm{h}, R=30 \mathrm{~m}, a=300 \mathrm{~m}, \beta_{T}=\beta_{R}=$ $\pi / 2, \alpha_{v}=\pi, N_{i}=64, D=300 \mathrm{~m}$, and $D=500 \mathrm{~m}$ for the obstructed and unobstructed segments, respectively. 


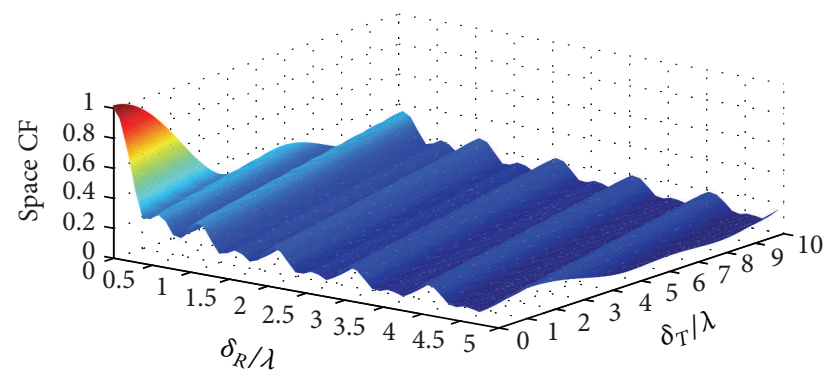

FIgURE 10: The space CF in the obstructed segment.

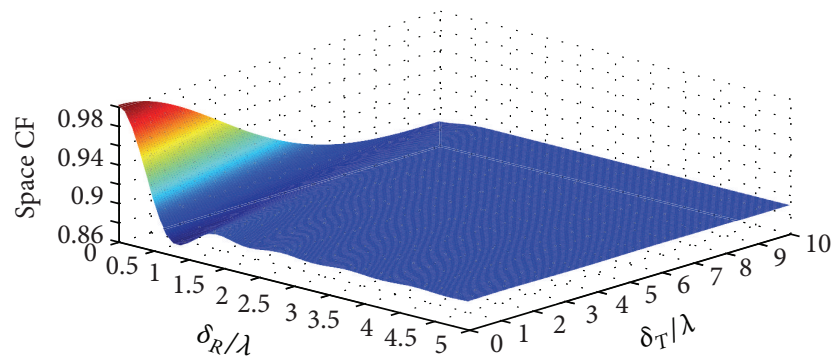

FIGURE 11: The space CF in the unobstructed segment.

Figures 10 and 11 illustrate the impact of the antenna element spacing on the CFs in the obstructed and unobstructed segments. It is obvious that the space correlation in the obstructed segment is lower than that in the unobstructed case. We also notice that the space correlation in the obstructed region decreases faster than that in the unobstructed situation when $\delta_{R}<1 \lambda$. And it has a larger fluctuation when $\delta_{R}>1 \lambda$.

Figure 12 depicts the time CFs for SISO $\left(\delta_{T}=\delta_{R}=0\right)$ and MIMO $\left(\delta_{T}=\delta_{R}=3 \lambda\right)$ cases in the obstructed and unobstructed segments. It is clear that there is a lower time correlation in the obstructed segment. The space separation leads to the significant decline of the time correlation in the obstructed segment, while it has little impact in the unobstructed region. By using the same parameters as in Figure 12, Figure 13 shows the corresponding (space-) Doppler PSDs. The space separation results in the fluctuations on the Doppler PSDs in both segments; however, it causes more dramatic fluctuation in the obstructed segment.

The ergodic capacities against SNR for the simulation model and Rayleigh model are compared in Figure 14. It is found that the SISO Rayleigh model and uncorrelated MIMO Rayleigh model have the lower and upper bounds of the capacity, respectively. The capacity for the MIMO case with $\delta_{T}=\delta_{R}=3 \lambda$ in the obstructed segment is very close to the upper bound, which is approximately $2 \mathrm{bits} / \mathrm{s} / \mathrm{Hz}$ higher than that in the unobstructed segment when SNR is $20 \mathrm{~dB}$. However, the capacity for the SISO case has the opposite variation tendency, which increases as the power of LOS components is enhanced.

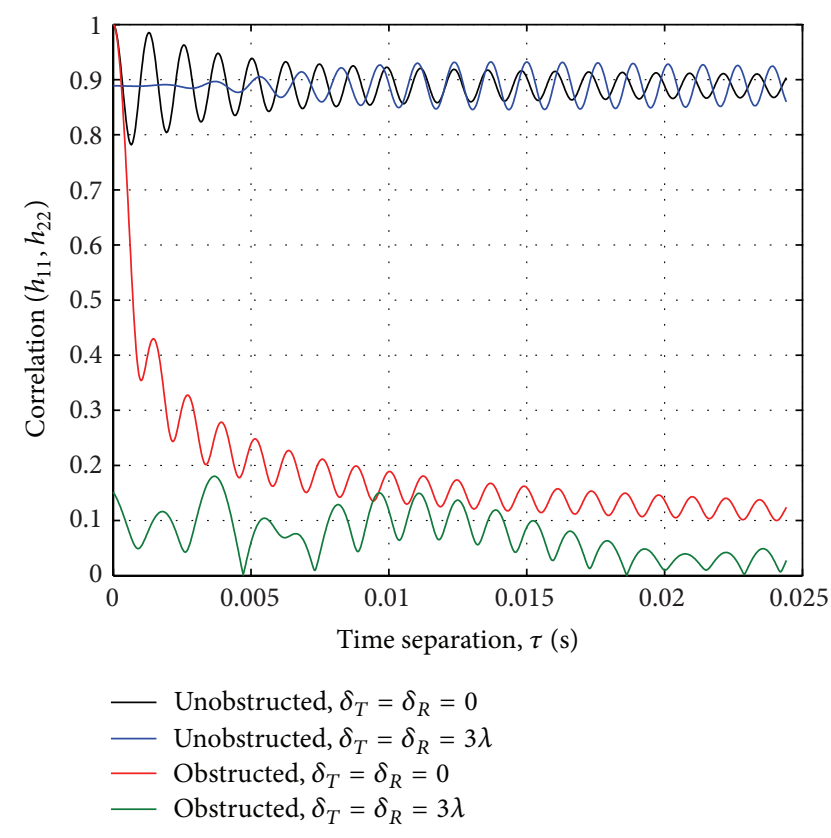

FIgure 12: Time CFs for SISO $\left(\delta_{T}=\delta_{R}=0\right)$ and $\operatorname{MIMO}\left(\delta_{T}=\delta_{R}=\right.$ $3 \lambda)$ cases in the obstructed and unobstructed segments.

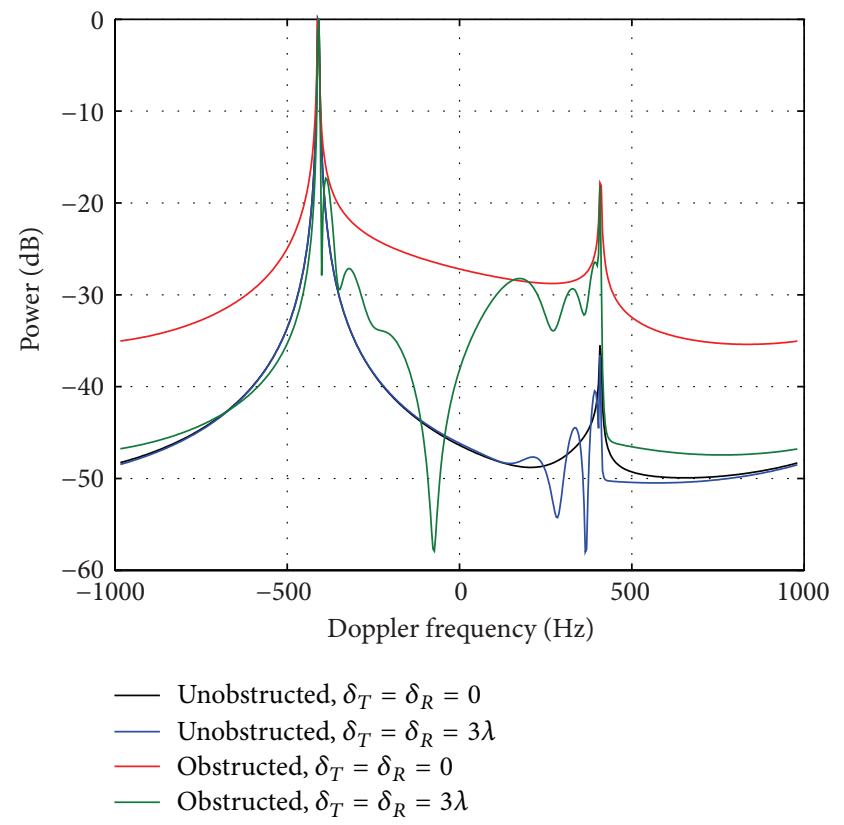

FIGURE 13: Doppler PSDs for SISO $\left(\delta_{T}=\delta_{R}=0\right)$ and $\operatorname{MIMO}\left(\delta_{T}=\right.$ $\left.\delta_{R}=3 \lambda\right)$ cases in the obstructed and unobstructed segments.

\section{Conclusion}

In this paper, we have proposed a semiempirical MIMO channel model for the HSR viaduct scenario. The empirical temporal fading properties including the $K$-factor and the Doppler PSD are derived from the wideband channel measurements on Zhengzhou-Xian HSR in China. The theoretical GBSM that consists of the one-ring model and 


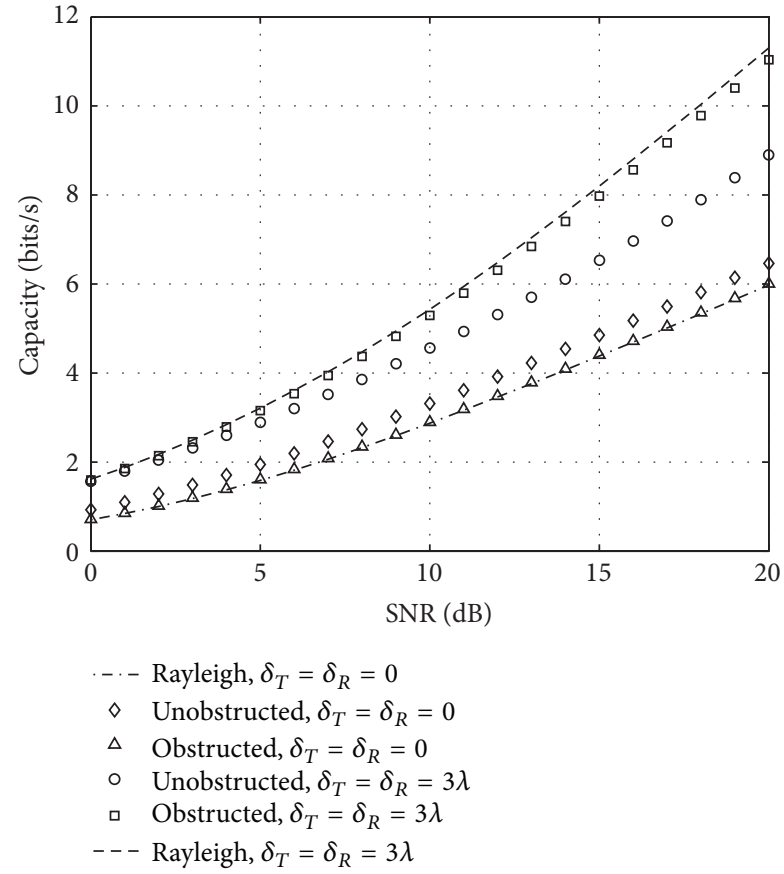

FIgURE 14: Ergodic capacities against SNR for SISO $\left(\delta_{T}=\delta_{R}=\right.$ $0)$ and MIMO $\left(\delta_{T}=\delta_{R}=3 \lambda\right)$ cases in the obstructed and unobstructed segments.

the elliptical model is developed to describe the whole propagation scenario that is partitioned into the obstructed segment and the unobstructed segment. By applying the estimated environment-related parameters to the GBSM, it has shown that the theoretical Doppler PSDs match the measured data very well. Then, a deterministic simulation model is established to analyze the ST CF, the SD PSD, and the channel capacity for the measured viaduct scenario. The results have demonstrated that the antenna element spacing has the larger impact on the ST CFs and SD PSDs in the obstructed segment. They have also shown that the capacity in the obstructed segment is about $2 \mathrm{bits} / \mathrm{s} / \mathrm{Hz}$ higher than that in the unobstructed region when the antenna element spacing is 3 wavelengths and SNR is $20 \mathrm{~dB}$.

\section{Conflict of Interests}

The authors declare that there is no conflict of interests regarding the publication of this paper.

\section{Acknowledgments}

The research was supported in part by the NSFC Project under Grants no. 61371070 and no. 61032002, the Open Research Fund of National Mobile Communications Research Laboratory, Southeast University, under Grant no. 2012D07, and the Fundamental Research Funds for the Central Universities under Grant no. 2013YJS016.

\section{References}

[1] 3GPP TS 36.104 V9.3.0, “Technical specification group radio access network; evolved universal terrestrial radio access; base station radio transmission and reception," 2010.

[2] P. Kyösti, "WINNER II channel models part II radio channel measurement and analysis results," Tech. Rep., 2007.

[3] P. Aikio, R. Gruber, and P. Vainikainen, "Wideband radio channel measurements for train tunnels," in Proceedings of the 48th IEEE Vehicular Technology Conference (VTC '98), pp. 460464, May 1998.

[4] R. He, Z. Zhong, B. Ai, and J. Ding, "Propagation measurements and analysis for high-speed railway cutting scenario," Electronics Letters, vol. 47, no. 21, pp. 1167-1168, 2011.

[5] R. He, Z. Zhong, B. Ai, and J. Ding, "An empirical path loss model and fading analysis for high-speed railway viaduct scenarios," IEEE Antennas and Wireless Propagation Letters, vol. 10, pp. 808-812, 2011.

[6] L. Liu, C. Tao, J. Qiu et al., "Position-based modeling for wireless channel on high-speed railway under a viaduct at $2.35 \mathrm{GHz}$," IEEE Journal on Selected Areas in Communications, vol. 30, no. 4, pp. 834-845, 2012.

[7] A. Ghazal, C.-X. Wang, H. Haas et al., "A non-stationary MIMO channel model for high-speed train communication systems," in Proceedings of the 75th IEEE Vehicular Technology Conference (VTC '12), June 2012.

[8] B. Chen and Z. Zhong, "Geometry-based stochastic modeling for MIMO channel in high-speed mobile scenario," International Journal of Antennas and Propagation, vol. 2012, Article ID 184682, 6 pages, 2012.

[9] L. Tian, X. Yin, Q. Zuo, J. Zhou, Z. Zhong, and S. X. Lu, "Channel modeling based on random propagation graphs for high speed railway scenarios," in Proceedings of the 23rd IEEE International Symposium on Personal, Indoor and Mobile Radio Communications (PIMRC '12), pp. 1746-1750, September 2012.

[10] Sencity Rail Antenna: 1399.17.0039 HUBER+SUHNER data sheet, HUBER+SUHNERAG RF Industrial, 2010.

[11] B. Ai, R. He, Z. Zhong et al., "Radio wave propagation scene partitioning for high-speed rails," International Journal of Antennas and Propagation, vol. 2012, Article ID 815232, 7 pages, 2012.

[12] D. Dong, J. Zhang, Y. Zhang, and X. Nie, "Large scale characteristics and capacity evaluation of outdoor relay channels at 2.35 GHz," in Proceedings of the 70th IEEE Vehicular Technology Conference Fall (VTC '09), September 2009.

[13] L. J. Greenstein, D. G. Michelson, and V. Erceg, "Momentmethod estimation of the Ricean K-factor," IEEE Communications Letters, vol. 3, no. 6, pp. 175-176, 1999.

[14] A. Abdi, J. A. Barger, and M. Kaveh, "A parametric model for the distribution of the angle of arrival and the associated correlation function and power spectrum at the mobile station," IEEE Transactions on Vehicular Technology, vol. 51, no. 3, pp. 425-434, 2002.

[15] M. Pätzold and B. O. Hogstad, "A space-time channel simulator for MIMO channels based on the geometrical one-ring scattering model," in Proceedings of the 60th IEEE Vehicular Technology Conference (VTC '04), pp. 144-149, September 2004.

[16] M. Pätzold and B. O. Hogstad, "A wideband MIMO channel model derived from the geometric elliptical scattering model," Wireless Communications and Mobile Computing, vol. 8, no. 5, pp. 597-605, 2008.

[17] X. Cheng, C.-X. Wang, D. I. Laurenson, S. Salous, and A. V. Vasilakos, "An adaptive geometry-based stochastic model 
for non-isotropic MIMO mobile-to-mobile channels," IEEE Transactions on Wireless Communications, vol. 8, no. 9, pp. 4824-4835, 2009.

[18] A. Abdi and M. Kaveh, "A space-time correlation model for multielement antenna systems in mobile fading channels," IEEE Journal on Selected Areas in Communications, vol. 20, no. 3, pp. 550-560, 2002.

[19] C. Gutiérrez-Díaz-de-León and M. Pätzold, "Sum-of-sinusoidbased simulation of flat fading wireless propagation channels under nonisotropic scattering conditions," in Proceedings of the IEEE Global Telecommunications Conference (GLOBECOM '07), pp. 3842-3846, November 2007. 

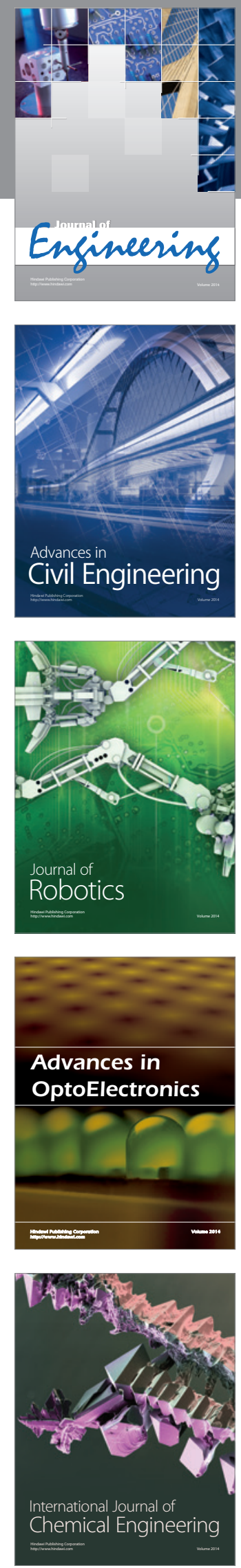

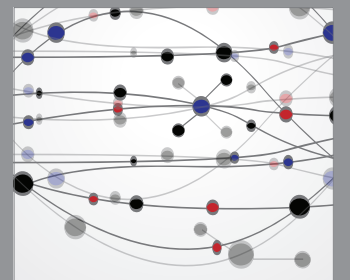

The Scientific World Journal
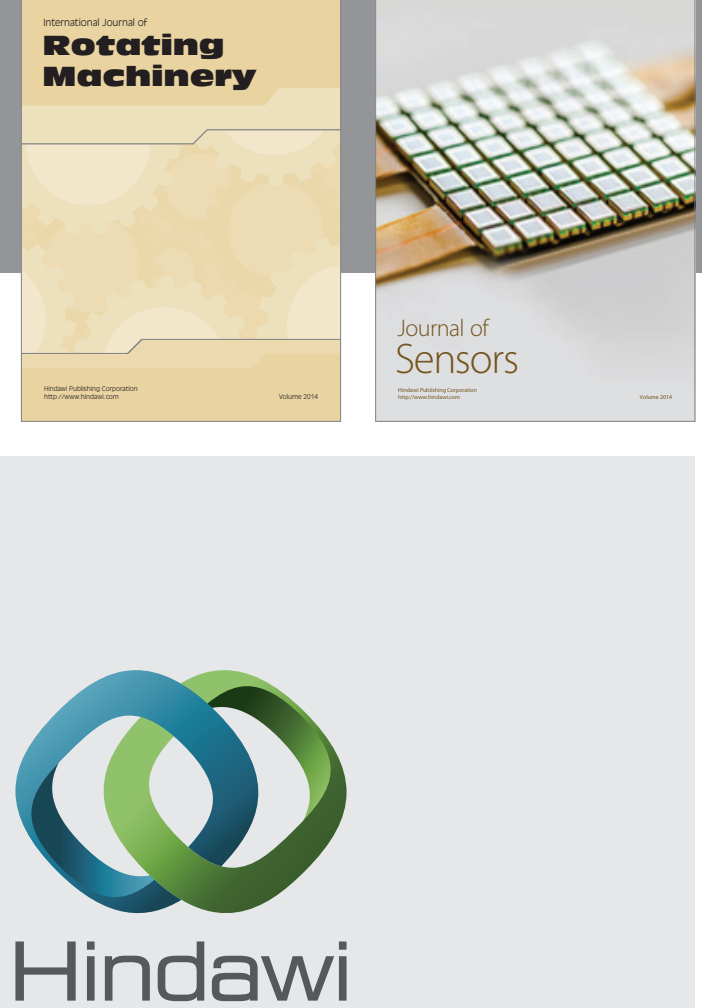

Submit your manuscripts at http://www.hindawi.com
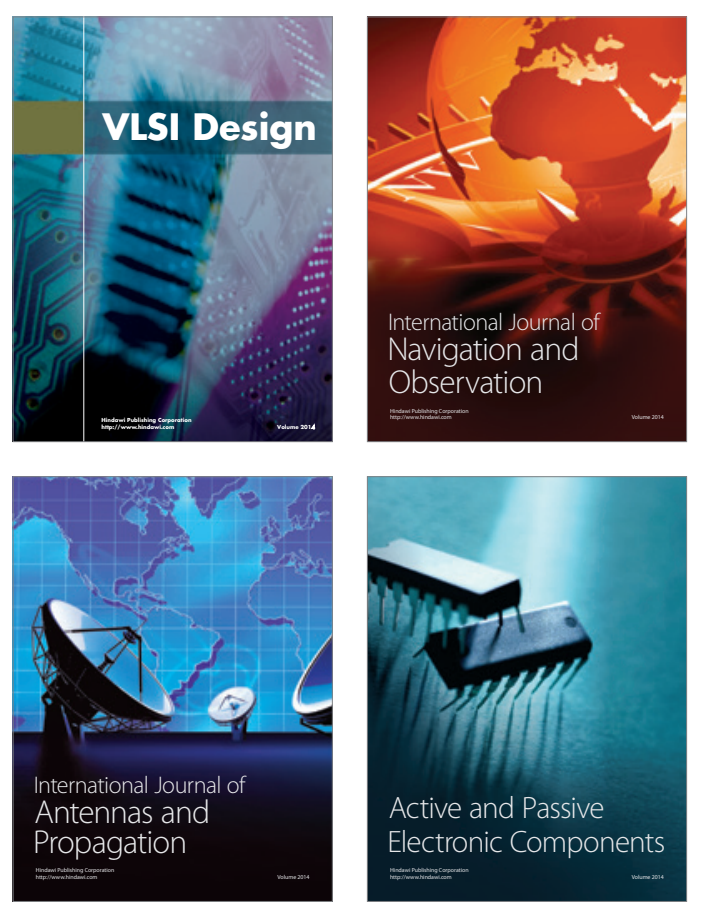
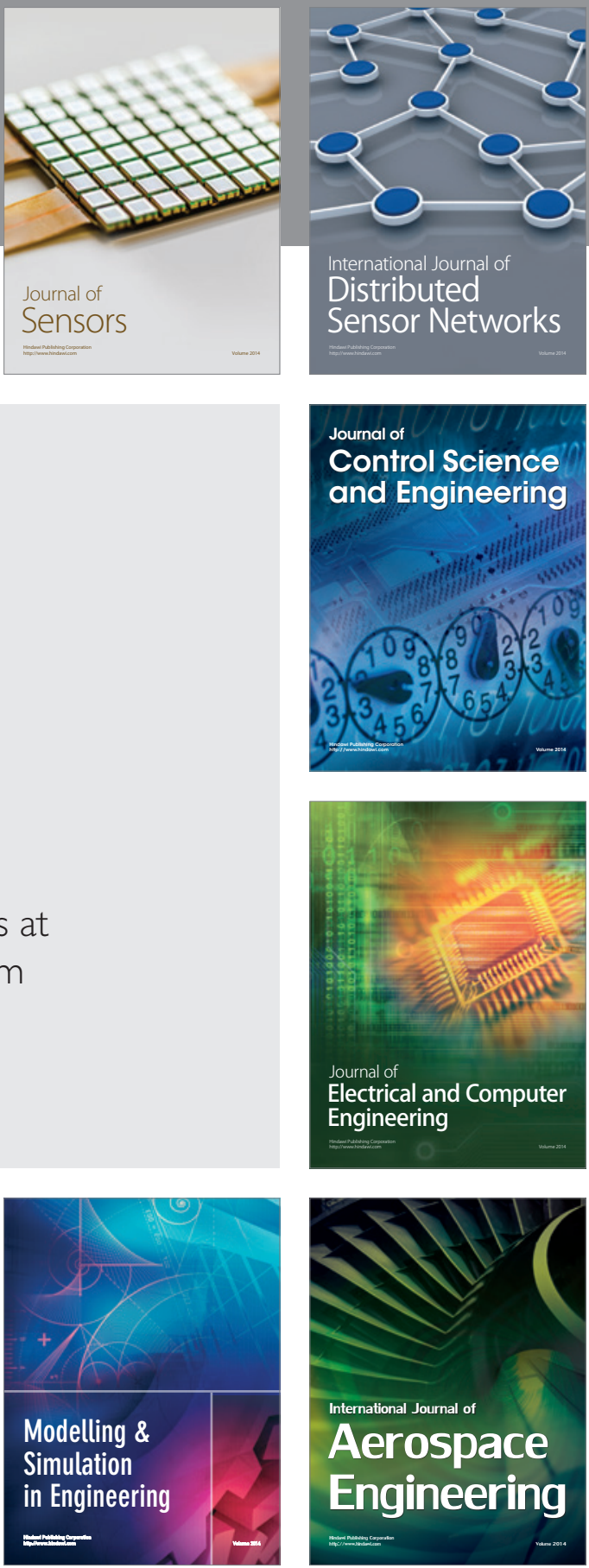

Journal of

Control Science

and Engineering
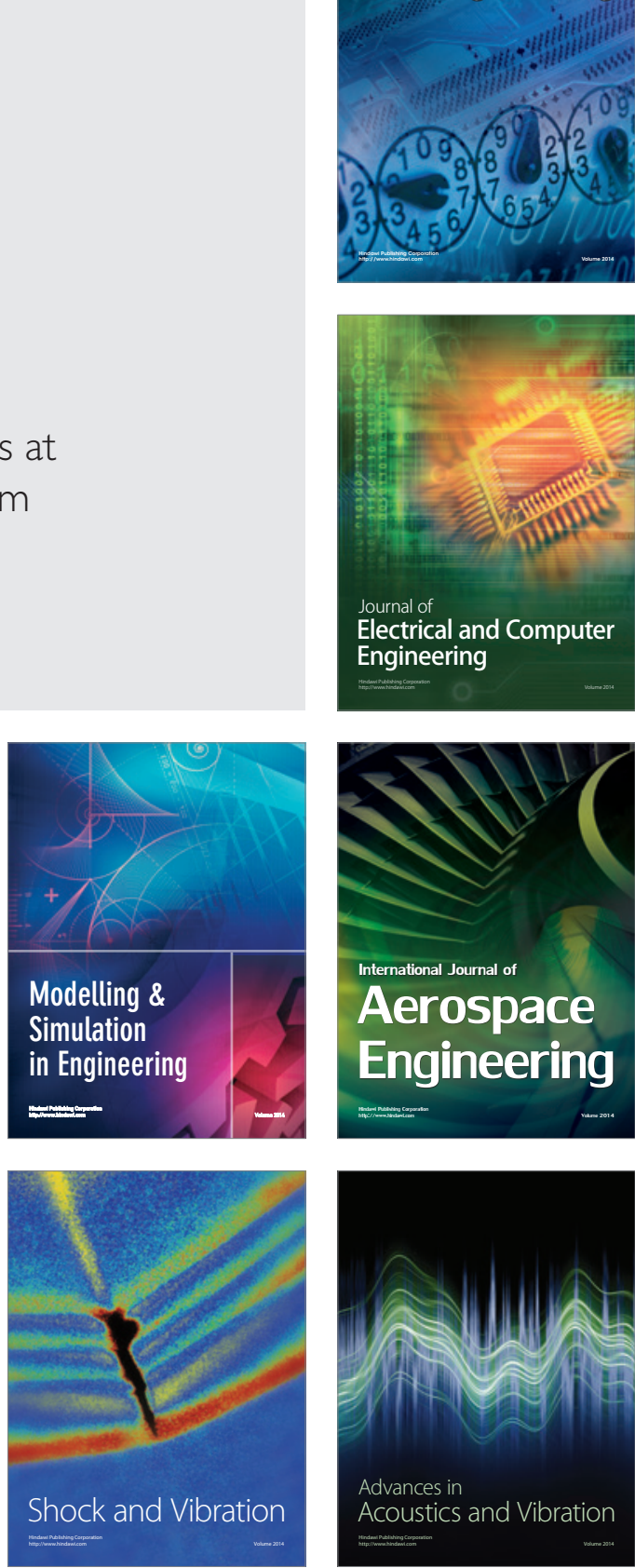